\title{
CROSSOVER FROM BCS TO BOSE-EINSTEIN IN HUBBARD MODEL
}

\author{
M.H. Pedersen, T. Schneider \\ IBM Research Division, Zurich Research Laboratory, Rüschlikon, Switzerland \\ AND H. BECK \\ Institut de Physique, Université Neuchâtel, Switzerland
}

\begin{abstract}
We generalize the standard attractive Hubbard, having an on-site pair creation operator $Q_{i}=a_{i \uparrow} a_{i \downarrow}$, to one with $n$ components $Q_{i \alpha}=a_{i \alpha \uparrow} a_{i \alpha \downarrow}, \alpha=$ $1,2, \ldots, n$. In the limit $n \rightarrow \infty$ we obtain the Ginzburg-Landau functional. On this basis we explore the crossover from weak (BCS) to strong coupling (Bose-Einstein condensation) superconductivity. The associated self-consistent equations for the Ginzburg-Landau parameters are similar to those of the $T$-matrix approach. The evolution of the band structure with increasing interaction strength is studied and correlated with the behavior of the pair propagator and the transition temperature. We find that the pairing interaction creates a new band which moves downwards in energy as the interaction strength increases and separates into a lower Hubbard band when the interaction strength becomes comparable to the band width. In the strong coupling regime, a third band with small spectral weight is also found in between the lower and upper Hubbard bands.
\end{abstract}

PACS numbers: 71.10.-w, 71.10.Fd, 71.27.+a, 74.20.-z

\section{Introduction}

The crossover from weak to strong coupling superconductivity in the attractive Hubbard model is a problem of considerable interest [1]. In the weak coupling regime considerable progress has been made using the $T$-matrix approach [2]. In particular, essential deviations from BCS-type behavior have been found. In the strong coupling limit, degenerate perturbation theory applies and superconductivity arises due to the condensation of preformed pairs. In the intermediate coupling regime, however, one has to rely on quantum Monte Carlo results which, unfortunately, are restricted to rather small systems [3]. In this paper we present a generalization of the attractive Hubbard model, which reduces to the spherical model [4] in the classical limit. In this model, treated with the functional integral technique, no uncontrolled approximation has to be invoked to calculate the properties of interest. Here we concentrate on the normal-state properties and in particular on the phase-transition line, i.e., the dependence of the transition temperature on interaction strength. 


\section{Formal matters}

The standard Hubbard model is generalized as follows. Introducing a pair creator with $n$ components, the Hamiltonian reads

$$
\mathcal{H}=\sum_{i j \sigma \alpha}\left(t_{i j}-\mu \delta_{i j}\right) c_{\alpha, i \sigma}^{\dagger} c_{\alpha, j \sigma}-\frac{U}{n} \sum_{i \alpha \alpha^{\prime}} Q_{i \alpha}^{\dagger} Q_{i \alpha^{\prime}}
$$

where $Q_{i \alpha}=a_{i \alpha \uparrow} a_{i \alpha \downarrow}, \alpha=1,2, \ldots, n, t_{i j}=t$ is the hopping between nearest neighbors and $\mu$ is the chemical potential. For $n=1$ the Hamiltonian reduces to the standard Hubbard model.

The interaction term is eliminated in terms of the Hubbard-Stratonovich identity for each time slice and site $[5,6]$

$$
\int \mathcal{D}^{2} \Delta(i, \tau) \exp \left(-\frac{n}{U}\left|\Delta(i, \tau)+\frac{U}{n} \sum_{\alpha} Q_{i \alpha}(\tau)\right|^{2}\right)=\text { const }
$$

yielding a Ginzburg-Landau theory

$$
\mathcal{Z}=\int \mathcal{D}^{2} \Delta \exp \left[n\left(\operatorname{Tr} \ln G_{0}-\operatorname{Tr} \ln \left(1-G_{0} \Delta\right)-\int_{0}^{\beta} \mathrm{d} \tau \sum_{i} \frac{|\Delta(i, \tau)|^{2}}{U}\right)\right]
$$

where

$$
\begin{aligned}
& G^{-1}=G_{0}^{-1}-\Delta, \\
& G_{0}^{-1}\left(i j, \tau \tau^{\prime}\right)=-\left(\partial_{\tau} I \delta_{i j}+t_{i j} \sigma_{z}-\mu \delta_{i j} \sigma_{z}\right) \delta\left(\tau-\tau^{\prime}\right), \\
& \Delta\left(i j, \tau \tau^{\prime}\right)=\left(\begin{array}{cc}
0 & \Delta_{i} \\
\Delta_{i}^{*} & 0
\end{array}\right) \delta_{i j} \delta\left(\tau-\tau^{\prime}\right) .
\end{aligned}
$$

Upon taking the limit $n \rightarrow \infty$ it can be shown that the Ginzburg-Landau functional becomes Gaussian in form [7]. The result is an effective action

$$
S[\Delta]=S_{0}-\operatorname{Tr} \ln G_{0}+\sum_{k, \mathrm{i} \omega_{n}}\left[\frac{1}{U}+\chi\left(k, \mathrm{i} \omega_{n}\right)\right]\left|\Delta\left(k, \mathrm{i} \omega_{n}\right)\right|^{2},
$$

where $\mathrm{i} \omega_{n}$ is a bosonic Matsubara frequency, $S_{0}$ - some constant, and the susceptibility, $\chi\left(k, \mathrm{i} \omega_{n}\right)$, is given by a set of self-consistent equations

$$
\begin{aligned}
& \chi\left(k, \mathrm{i} \omega_{n}\right)=-\sum_{q, \mathrm{i} \epsilon_{n}} G\left(q, \mathrm{i} \epsilon_{n}\right) G_{0}\left(k-q, \mathrm{i} \omega_{n}-\mathrm{i} \epsilon_{n}\right), \\
& G^{-1}\left(k, \mathrm{i} \epsilon_{n}\right)=G_{0}^{-1}\left(k, \mathrm{i} \epsilon_{n}\right)+\Sigma\left(k, \mathrm{i} \epsilon_{n}\right), \\
& \Sigma\left(k, \mathrm{i} \epsilon_{n}\right)=\sum_{q, \mathrm{i} \omega_{n}} G_{0}\left(q-k, \mathrm{i} \omega_{n}-\mathrm{i} \epsilon_{n}\right)\left\langle\left|\Delta\left(q, \mathrm{i} \omega_{n}\right)\right|^{2}\right\rangle, \\
& \left\langle\left|\Delta\left(q, \mathrm{i} \omega_{n}\right)\right|^{2}\right\rangle=\frac{U}{1+U \chi\left(q, \mathrm{i} \omega_{n}\right)}
\end{aligned}
$$

and $i \epsilon_{n}$ is a fermionic Matsubara frequency.

Figure 1 shows diagrams of the self-consistent equations, with the notation $T(k, z)=\left\langle|\Delta(k, z)|^{2}\right\rangle$. Interestingly, the structure of the equations is very similar 


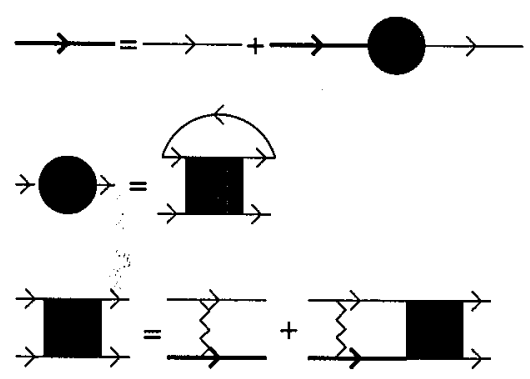

Fig. 1. Diagrammatic representation of the large- $n$ self-consistent equations. Bold lines are dressed Green's functions and thin lines are bare ones.

to the $T$-matrix equations obtained by an infinite resummation of ladder diagrams [2]. It should be noted that in the present approach not all internal lines are dressed, which is an important difference between the conventional approach and the present formalism. In the limit $t \rightarrow 0$ we recover correctly the atomic limit, whereas the standard $T$-matrix approach fails to do so. Indeed, a quick calculation for small densities, $\rho$, shows that the set

$$
\begin{aligned}
& G(z)=\frac{\rho}{z+\mu+U}+\frac{1-\rho}{z+\mu}, \\
& G_{0}(z)=\frac{1}{z+\mu+\rho U}
\end{aligned}
$$

is a self-consistent solution, where $G(z)$ is the exact result in this limit. Thus, our equation seems to be clearly superior to the conventional approach.

\section{Numerical results}

A numerical solution of the self-consistent set of equations has been made in three dimensions using a Fourier technique. A particularly interesting result is the evolution of the transition temperature as a function of interaction strength, $U$. As seen from the Ginzburg-Landau functional the transition occurs when $1 / U+$ $\chi(0,0)=0$. In Fig. 2 we show results of solving this equation for a number of interaction strengths at a fixed filling fraction of $\rho=0.2$. For small $U$ the transition temperature rises exponentially as expected from BCS, but the numerical results do not seem to go to the BCS curve (solid line) for finite $U$. This effect is probably partially due to finite-size effects because transition temperatures were obtained only for a $8 \times 8 \times 8$ system. For temperatures above approximately $0.15 t$ no significant finite-size effects were observed compared to $16 \times 16 \times 16$ systems, but for low temperatures finite-size effects become increasingly important. For large $U$ the transition temperature is expected to decrease as $t^{2} / U$, because the Hubbard model can be mapped to a model of hard-core bosons with a hopping due to pair dissociation in this limit [8]. This behavior is also corroborated by our results.

The evolution of the electronic state with increasing interaction is revealed by observing the pair propagator, $T(k, z)$. For small $U$ and small momenta the structure of the propagator is that of a resonance, and the phase transition is BCS-like. For large momenta a bound state is observed in the propagator. This 


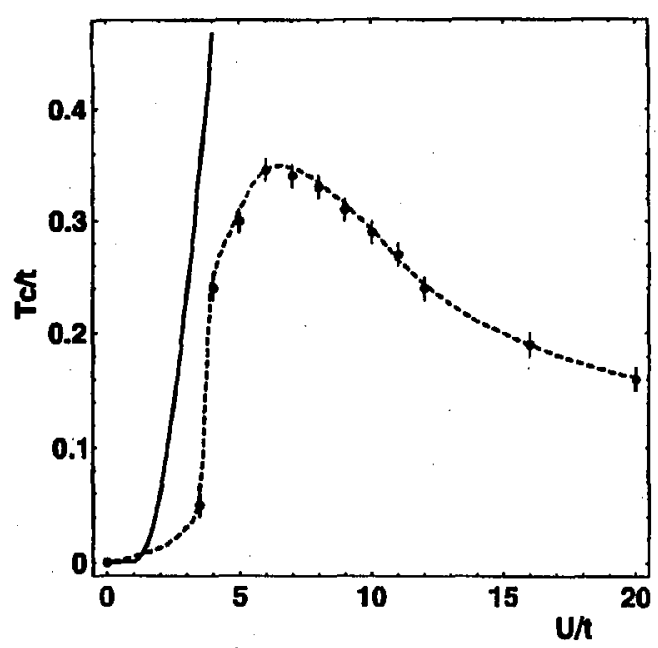

Fig. 2. Transition temperature as a function of interaction strength, both in units of $t$. Results are for an $8 \times 8 \times 8$ system at a filling fraction of $\rho=0.2$; error bars are estimates. The dashed curve is a guide to the eye and the solid curve is the result of solving the BCS gap equation.

bound state creates a new band of paired electrons which intersects the free electron band, hybridizes it, and yields two bands. For increasing $U$ the bound state is observed for smaller momenta and when $U$ becomes comparable to the free band width, $12 t$, there is a bound state for all momenta. The phase transition is now a Bose-Einstein condensation of local pairs. The band of bound electrons will now have moved below the free band and we observe a lower IIubbard band of paired electrons and an upper Hubbard band of free electrons. In between these two bands a small third band is observed which can be attributed to the coupling of an electron to a sound-like mode of the quantum liquid.

\section{Acknowledgments}

We thank Roman Micnas and Johannes Singer for useful discussions.

\section{References}

[1] C.A.R. Sa de Melo, M. Randeria, J.R. Engelbrecht, Phys. Rev. Lett. 71, 3202 (1993).

[2] R. Micnas, M.H. Pedersen, S. Schafroth, T. Schneider, J.J. Rodríguez-Nũnez, H. Beck, Phys. Rev. B 52, 16223 (1996).

[3] J.M. Singer, M.H. Pedersen, T. Schneider, Phys. Rev. B, 1996, in press.

[4] T.H. Berlin, M. Kac, Phys. Rev. 86, 821 (1952).

[5]. N. Read, D.M. Newns, J. Phys. C 16, 3273 (1983).

[6] A. Auerbach, Interacting Electrons and Quantum Magnetism, Springer-Verlag, Berlin 1994.

[7] M.H. Pedersen, T. Schneider, H. Beck, in preparation.

[8] R. Micnas, J. Ranninger, S. Robaszkiewicz, Rev. Mod. Phys. 62, 113 (1990). 